\title{
Epitaxial Thin Films on Tsai-type Quasicrystals
}

\author{
H. R. Sharma, S. Coates, S. Hars, and R. McGrath \\ The University of Liverpool, Department of Physics, Liverpool, UK
}

h.r.sharma@liv.ac.uk

We will present several interesting structures of thin films grown on Tsai-type quasicrystal, icosahedral (i)-Ag-In-Yb, studied by various experimental techniques including scanning tunnelling microscopy (STM). The results include three dimensional quasicrystalline films of single elements [1] and molecular films [2] (Figure 1).

The $i$-Ag-In- $\mathrm{Yb}$ quasicrystal is built by rhombic triacontahedral (RTH) clusters and its surface is formed at the bulk atomic planes that bisect the RTH clusters [3]. When $\mathrm{Pb}$ is deposited on the fivefold $i$ - $\mathrm{Ag}$-In- $\mathrm{Yb}$ surface, the $\mathrm{Pb}$ atoms adsorb at the sites that were originally occupied by the cluster atoms and thus produce quasicrystalline film in three-dimension [1]. This observation is evidenced in other systems as well, namely $\mathrm{Pb}$ on the threefold and twofold $i$-Ag-In-Yb surfaces [4,5] and In, $\mathrm{Sb}$ and $\mathrm{Bi}$ on the fivefold $i$-Ag-In$\mathrm{Yb}$ surface [6].

We also found that Pentacene molecules deposited on the fivefold $i$-Ag-In- $\mathrm{Yb}$ surface adsorb at tenfold-symmetric sites of $\mathrm{Yb}$ atoms around surface-bisected RTH clusters, yielding quasicrystalline order [2]. The selective adsorption of Pentacene on Yb sites is also observed on the threefold and twofold surfaces of the same sample.

The phenomena of adsorption on selective sites is also found on Al-based quasicrystals. $\mathrm{C}_{60}$ molecules preferably adsorb on Fe or Mn when deposited on surfaces of $i$-Al-Pd-Mn and $i$-Al-Cu-Fe $[2,7]$, yielding quasicrystalline order of $\mathrm{C}_{60}$. The compatibility between the characteristic lengths of the substrate and the size of adsorbates has led to the growth of unprecedented epitaxial structures.

(a)

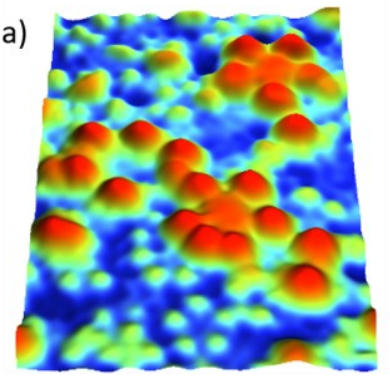

(b)

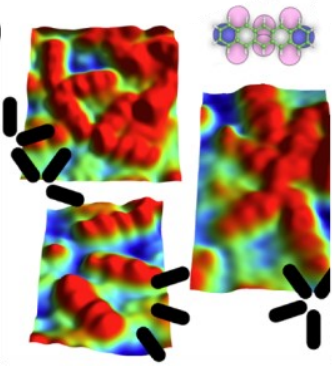

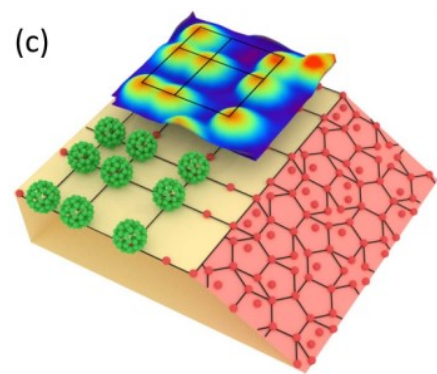

Figure 1. (a) $\mathrm{Pb}$ on the fivefold $i$-Ag-In- $\mathrm{Yb}$ surface [1], (b) Pentacene on the fivefold $i$-Ag-In- $\mathrm{Yb}$ surface [2], and (c) $\mathrm{C}_{60}$ on the twofold $i$-Ag-In- $\mathrm{Yb}[7]$.

[1] Sharma, H. R., Nozawa, K., Smerdon, J. A., Nugent, P.J., McLeod, I., Dhanak, V. R., Shimoda, M., Ishii, Y., Tsai, A. P., \& McGrath R. (2013). Nature Communications 4, 2715.

[2] Smerdon, J. A., Young, K., Lowe, M., Hars, S. S., Yadav, T. P., Hesp, D., Dhanak, V. R., Tsai, A. P., Sharma, H. R. \& McGrath R. (2014). Nano Letters 14, 1184.

[3] Sharma, H. R., Shimoda, M., Sagisaka, K., Takakura, H., Smerdon, J. A., Nugent, P. J., McGrath, R., Fujita, D., Ohhashi, S. \& Tsai, A. P. (2009). Physical Review B 80, 121401 (R).

[4] Coates, S., McGrath R. \& Sharma, H. R. (2020). Journal of Physics: Condensed Matter 32, 425006.

[5] Coates, S., Thorn, S., McGrath, R., Sharma, H. R. \& Tsai, A. P. (2020). Physical Review Materials 4, 026003.

[6] Hars, S. S., Sharma, H. R., Smerdon, J. A., Coates, S., Nozawa, K., Tsai, A. P. \& McGrath, R. (2018). Surface Science 678, 222.

[7] Coates, S., Smerdon, J. A., McGrath, R. \& Sharma, H. R. (2018). Nature Communications 9, 3435.

Keywords: Quasicrystals; Thin Films; $\mathrm{C}_{60}$, Pentacene, Scanning Tunnelling Microscope 